\title{
Structural study of helical polyfluorene under high quasihydrostatic pressure
}

\author{
M. Knaapila, ${ }^{1,}{ }^{*}$ Z. Konôpková, ${ }^{2}$ M. Torkkeli, ${ }^{3}$ D. Haase, ${ }^{4}$ H.-P. Liermann, ${ }^{2}$ S. Guha, ${ }^{5}$ and U. Scherf ${ }^{6}$ \\ ${ }^{1}$ Physics Department, Institute for Energy Technology, Kjeller NO-2027, Norway \\ ${ }^{2}$ DESY Photon Science, Hamburg D-22607, Germany \\ ${ }^{3}$ Department of Physics, University of Helsinki, Helsinki FI-00014, Finland \\ ${ }^{4}$ MAX IV-Laboratory, Lund University, Lund SE-22100, Sweden \\ ${ }^{5}$ Department of Physics and Astronomy, University of Missouri, Columbia, Missouri 65211, USA \\ ${ }^{6}$ Macromolecular Chemistry Group, Bergische Universität Wuppertal, Wuppertal D-42119, Germany \\ (Received 5 October 2012; revised manuscript received 27 December 2012; published 19 February 2013)
}

\begin{abstract}
We report on an x-ray diffraction (XRD) study of helical poly[9,9-bis(2-ethylhexyl)fluorene] (PF2/6) under high quasihydrostatic pressure and show an effect of pressure on the torsion angle (dihedral angle) between adjunct repeat units and on the hexagonal unit cell. A model for helical backbone conformation is constructed. The theoretical position for the most prominent $00 l \mathrm{x}$-ray reflection is calculated as a function of torsion angle. The XRD of high molecular weight PF2 $/ 6\left(M_{\mathrm{n}}=30 \mathrm{~kg} / \mathrm{mol}\right)$ is measured through a diamond anvil cell upon pressure increase from 1 to $10 \mathrm{GPa}$. The theoretically considered $00 l$ reflection is experimentally identified, and its shift with the increasing pressure is found to be consistent with the decreasing torsion angle between 2 and $6 \mathrm{GPa}$. This indicates partial backbone planarization towards a more open helical structure. The $h 00$ peak is identified, and its shift together with the broadening of $00 \mathrm{l}$ implies impairment of the ambient hexagonal order, which begins at or below $2 \mathrm{GPa}$. Previously collected high-pressure photoluminescence data are reanalyzed and are found to be qualitatively consistent with the XRD data. This paper provides an example of how the helical $\pi$-conjugated backbone structure can be controlled by applying high quasihydrostatic pressure without modifications in its chemical structure. Moreover, it paves the way for wider use of high-pressure x-ray scattering in the research of $\pi$-conjugated polymers.
\end{abstract}

DOI: 10.1103/PhysRevE.87.022602

PACS number(s): 36.20.-r, 62.50.-p, 61.05.cf

\section{INTRODUCTION}

Electronic properties of $\pi$-conjugated polymers originate from their intramolecular configuration, which largely depends on the intermolecular interactions. Here, an essential factor is the backbone conformation, which can be controlled by modifying polymer side chains as shown for archetypical polyfluorenes [1] or by incorporating new units into the polymer main chain in terms of copolymerization as shown for archetypical polythiophenes [2]. In an alternative strategy, intercalates or dopants are incorporated into the solid state structure, and the backbone rotation changes through the changed intermolecular environment as detailed early for poly ( $p$-phenylene vinylene) (PPV) [3]. Vapor treatment is another procedure for selecting a preferred phase among the ones with various degrees of planarity. This technique is well known, for example, when synthesizing the nearly planar $\beta$ phase of polycrystalline poly(9,9-dioctylfluorene) (PF8) [4]. Although these approaches are exceedingly important for the materials science and organic electronics, the effect of changing backbone conformation may not be isolated from the effect of changing the chemical environment.

Hydrostatic pressure is an elegant tool to enhance the intermolecular interactions in $\pi$-conjugated polymers and to influence their molecular geometry without chemical changes either in polymers or in their environments [5]. The high-pressure studies are rare, but there are several examples where increasing pressure leads to a redshift in

\footnotetext{
*Author to whom correspondence should be addressed: matti.knaapila@ife.no
}

the photoluminescence (PL) spectrum, which is, in turn, indirect evidence for the backbone planarization. In a prime example, Schmidtke and co-workers [6-8] studied poly(9,9dioctylfluorene-co-benzothiadiazole) (F8BT) using a variety of optical spectroscopy methods and increased pressure under quasihydrostatic conditions up to $8.8 \mathrm{GPa}$. The authors found rich optical effects, including a redshift in PL [6,7] and increased delocalization of photogenerated singlet excitons at high pressures [8]. They proposed that these effects have structural origins, involving reduction in the torsion angle (dihedral angle) between F8 and BT subunits. Similar effects were observed when these studies were extended to F8BT blends [9]. Morandi et al. [10] studied PPV up to $5 \mathrm{GPa}$ and reported a bathochromic shift in $\pi-\pi *$ transition explained by the enhanced intermolecular interactions. Paudel et al. $[11,12]$ studied a variety of polyfluorenes, including PF8, and observed a redshift in the PL spectrum also explained by the higher conjugation and supposed planarization of the main chain.

The structural evidence provided by the optical studies should be confirmed and should be complemented by direct structural data obtained by means of powder x-ray diffraction (XRD), but this research line remains largely unexplored for a variety of reasons. Perhaps, the only feasible probe is a powerful synchrotron generated $\mathrm{x}$-ray beam passing through the diamond anvil cell (DAC), but as the diamond windows are an order of magnitude thicker than the samples themselves, they generate overwhelming Compton background and a poor signal-to-noise ratio. This is a smaller problem for inorganic materials [13], $\pi$-conjugated oligomers, such as oligo( $p$-phenylenes) [14], or their molecular counterparts, such as anthracene [15] or fluorene molecules [16], whose 


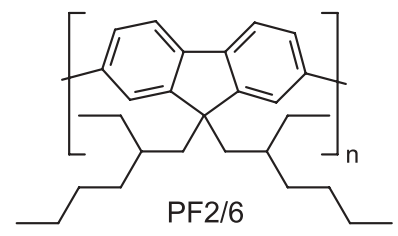

FIG. 1. Chemical structure of the PF2/6 polymer.

crystallites are regularly displaying strong Bragg reflections and a reasonable intensity. However, as the order in polymers is much weaker, the detection of XRD through the DAC becomes a nontrivial task.

In pilot papers, Mårdalen et al. [17,18] studied polythiophenes by means of powder XRD while increasing the quasihydrostatic pressure up to $10 \mathrm{GPa}$. The authors showed that the distance between neighboring chains decreased and the planarity of lamellar poly(3-octylthiophene) increased with increasing pressure and that this was related to a pressure induced optical transition where the material changed its color from red to yellow. They showed, moreover, that poly (3,3'-dioctyl-2,2'-bithiophene) experiences a reduction in the chain twist angle at high pressures, which explains a similar optical effect. The polythiophenes studied by Mårdalen et al. $[17,18]$ are lamellar, indicating that their packing density is related to the side chain interdigitation with increasing pressure. This was shown earlier by Corish et al. [19], who found, computationally, that the interlamellar cell parameter $a$ manifested several energy minima when the degree of interdigitation was enhanced by increasing pressure.

We propose that poly[9,9-bis(2-ethylhexyl)fluorene] (PF2/6) [20] shown in Fig. 1 would be an ideal nonlamellar candidate for consolidating optical and structural high-pressure investigations. The branched side chains make PF2/6 helical [21,22], and the torsion angles in vacuo are close to $\sim 145^{\circ}$ [23]. In the solid state, the helical chains form ensembles of three within hexagonal (Hex) unit cells that turn to the nematic (Nem) phase with increasing temperature or decreasing molecular weight [24]. Guha and Chandrasekhar [25] and Martin et al. [26] carried out optical high-pressure studies and reported that, when the hydrostatic pressure exceeds 2-4 GPa, the polymer shows enhancement in PL emission at 2.1-2.6 eV. This contribution stems from aggregates $(\sim 2.4 \mathrm{eV})$ and keto defects $(\sim 2.3 \mathrm{eV})$ and becomes dominant due to enhanced intermolecular interactions due to increasing pressure. The Raman peaks were found to harden with increasing pressure. The authors also indicated that the changes in the relative PL intensities resemble those expected for a crystalline-liquid-crystalline phase transition also known as the Hex-Nem transition in the terminology of Ref. [24]. These findings encouraged us to perform wide-angle $\mathrm{x}$-ray scattering experiments of PF2/6 at elevated pressures. The obtained data pointed to the changes in the intermolecular structure, beginning between 2 and $4 \mathrm{GPa}$, interpreted by packing arguments [27]. These changes seemed to coexist with the optically observed ones with speculated similarity with the ambient Hex-Nem phase transition. However, so far, no data allow the unambiguous interpretation of the main chain conformation.
In this paper, we show how the planarity and phase behavior of a $\pi$-conjugated polymer can be controlled by high quasihydrostatic pressure. Although this area has been pioneered by Schmidtke and others using optical methods, we provide direct structural powder XRD data interpreted against the molecular model and some reanalyzed PL data. We use PF2/6 as a standard for the helical $\pi$-conjugated polymer and make a connection between the molecular model and the experimental data of the helix structure in the intramolecular level. Furthermore, this paper contributes to extending the experimental playground of high-pressure XRD. We believe that the continuous efforts for developing these procedures will eventually open completely new possibilities for the researchers of $\pi$-conjugated polymers and polyfluorenes, in particular.

\section{THEORY}

We first place attention on the theoretically predicted XRD pattern as a function of torsion angle. It is experimentally found that the solid state PF2/6 adopts a 5-helical [28] or 21-helical main chain conformation at the ambient conditions [21]. The main chain is stiff with the persistence length of about $100 \AA$, and PF2/6 is generally interpreted as a hairy-rod type polymer [24].

At room temperature, helical chains can manifest a Hex phase, which consists of ensembles of three chains. The HexNem transition takes place with increasing temperature or/and with decreasing molecular weight [24]. The possibility for the Hex phase separates the high molecular weight (HMW) PF2/6 from the low molecular weight (LMW) PF2/6. This also means that there are actually two different Nem phases, one for HMW-PF2/6 and one for LMW-PF2/6. The former one exists above both the glass transition and the Hex-Nem transition and reveals, rather, a packing of individual chains, whereas, the latter one maintains the correlation of three chain bundles. The former one is less dense than the Hex phase, whereas, the latter one is marginally denser. The intermolecular structure is essentially maintained if the Nem LMW-PF2/6 is cooled down to the glass transition temperature, and we have called this structure the "frozen" or "glassy" Nem phase [24].

The glass transition of poly( $p$-phenylene) with sulfonated ester and dodecyl chains is increased with increasing pressure (when studied up to $200 \mathrm{MPa}$ ), and the high -pressure structure is brittle rather than flowable [29]. This may also be true for $\mathrm{PF} 2 / 6$, which is a closely related hairy-rod polymer. Therefore, we propose that its weakly ordered high-pressure structures should be compared to the ambient glassy Nem phase.

The helical structure is indicated by the prominent Bragg reflection along the (rodlike) chain axis, which is selected as the crystalline $\mathbf{c}$ axis. At ambient conditions, this reflection appears at $0.78 \AA^{-1}$ and corresponds to the monomer repeat (projected length of the monomer on the c axis). For a 21-helical chain, then, the reflection is indexed as 0021. The hexagonal structure can be followed by corresponding Bragg reflections in the plane perpendicular to the chain axis, spanned by the $\mathbf{a}$ and $\mathbf{b}$ axes (vide infra).

PF2/6 shows significant changes in PL and associated vibronics when hydrostatic pressure is increased up to $2 \mathrm{GPa}$ in bulk or $3.5 \mathrm{GPa}$ in thin films $[25,26]$. These optical changes 
must relate to the structural changes and could be explained by the partial planarization of the helical main chain and/or changes in the intermolecular level, observable by means of powder XRD.

If the hexagonal structure turns nematic at the ambient pressure, the reflection 0021 is present, but the peak width increases due to the loss of chain registry [24]. The reflections along the $\mathbf{a}$ and $\mathbf{b}$ axes become blurred halo type features.

If the helical structure was preserved but was slightly changed under high hydrostatic pressure, the structure would still lead to a strong $00 l$ reflection appearing near but not in exactly the same position as the 0021 reflection at ambient conditions. For any assumed helical structure, we may calculate the position of this reflection, but this solution is not unique, i.e., if the reflection is experimentally observed, we cannot exclude all hypothetical nonhelical structures.

The helical structure of PF2/6 is quite distinctive from the linear side chain polyfluorenes, such as PF6 [30] or PF8 [31]. The linear side chain polyfluorenes adopt a rather planar backbone in their crystalline structures, which typically manifest a repeat distance of four monomers. The so called $\beta$ phase is the most planar form and manifests the periodicity of two monomer units [31]. A reasonable model for this polymer class would feature all trans or near-trans torsion angles while keeping in mind that the fully trans conformation is energetically unfavorable (to the point of being sterically hindered). In this respect, it is instructive to consider chain geometries that lead to fourfold or twofold periodicity, which we illustrate in Fig. 2.

The first two geometries correspond to the trivial cases of regular $4 / 1$ and $2 / 1$ helices. The third alternative, designated

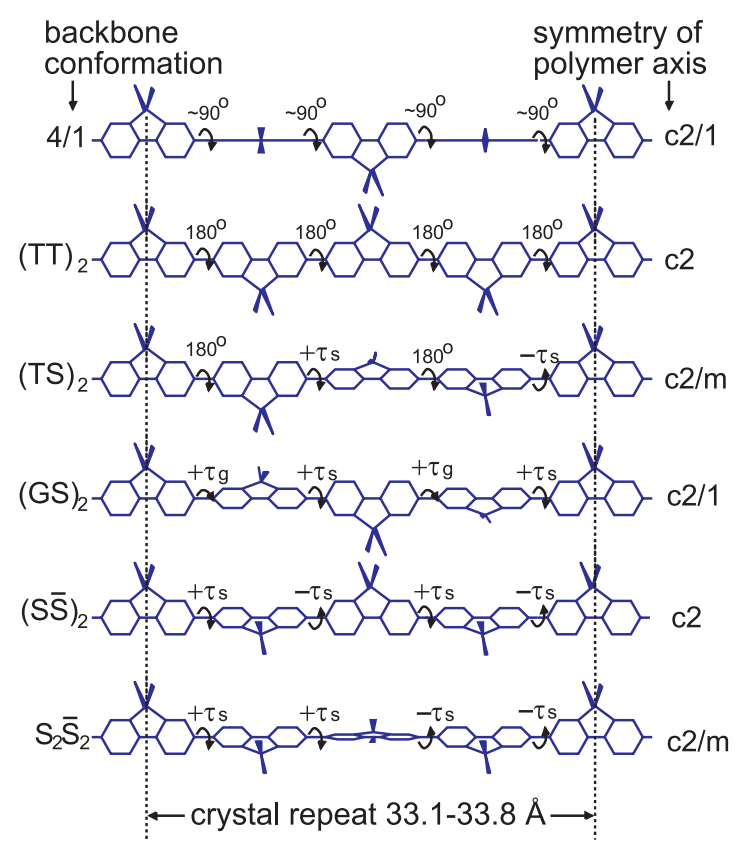

FIG. 2. (Color online) Models for the polyfluorene backbone conformations, which manifest different symmetries about the $\mathbf{c}$ axis and which produce the $00 l$ reflections. The models are characterized by torsion angles $\sim 90^{\circ}, 180^{\circ}, \pm \tau_{\mathrm{s}}$, and $\pm \tau_{\mathrm{g}} . T=$ trans; $S=$ skew; $G=$ gauche. Side chains are marked by single sticks for clarity. See text for details. as $(T S)_{2}$, has alternating trans and near-trans torsion angles. The trans bond produces a unit of two monomers that have parallel bonds to the neighboring units. Thus, the torsion angle $\tau_{\mathrm{s}}$ is freely variable and produces a periodic structure with alternating directions of rotation of the successive non trans bond. This structure, however, has the unfavorable trans bond. The fourth alternative $(G S)_{2}$ circumvents this by introducing two rotations that put the third monomer in the trans position to the first. The two rotations again, have 1 degree of freedom and satisfy $\tau_{\mathrm{s}}+\tau_{\mathrm{g}} \sim 180^{\circ}$. Thus, one of the rotations is always skew or nearly trans $(S)$ and the other is gauche or nearly cis $(G)$. The order and direction of the rotations is not strict, and we may have four alternative sequences in this family, which produce the fourfold periodicity $\left(\tau_{\mathrm{g}}, \tau_{\mathrm{s}}, \tau_{\mathrm{g}}, \tau_{\mathrm{s}}\right.$; $\tau_{\mathrm{g}}, \tau_{\mathrm{s}}, \tau_{\mathrm{s}}, \tau_{\mathrm{g}} ; \tau_{\mathrm{g}}, \tau_{\mathrm{s}},-\tau_{\mathrm{g}},-\tau_{\mathrm{s}}$; and $\left.\tau_{\mathrm{g}}, \tau_{\mathrm{s}},-\tau_{\mathrm{s}},-\tau_{\mathrm{g}}\right)$. For illustration, we have selected the one with the twofold screw symmetry axis. The two last cases in Fig. 2 represent twisted backbone conformations obtained by alternating left-hand and right-hand rotations $\tau_{\mathrm{s}}$ and $-\tau_{\mathrm{s}}$. These do not yield strict twofold or fourfold periodicities unless a slight deviation to the direct $\mathrm{C}-\mathrm{C}$ bond between monomers is allowed.

Figure 3 shows calculated monomer periods (as the corresponding peak positions) for diverse helical structures (dotted black curve), the $(G S)_{2}$ (solid blue curve), and the $(T S)_{2}$ forms (dashed red curve). In this calculation, we assume rigid monomers and fixed bond angles. The bond distances are based on the known $n$-octyl fluorene monomer structure according to McFarlane et al. [32]. The monomer repeat depends on the bond angle between fluorene moieties, which results from the monomer geometry assuming a direct bond between monomers. For the assumed bond angle of $11.43^{\circ}$, the $21 / 4$ helix is realized with a torsion angle of $65.09^{\circ}$, and the resulting monomer repeat $8.06 \AA$ is in good agreement with the experimental data [21]. The depicted helices do not

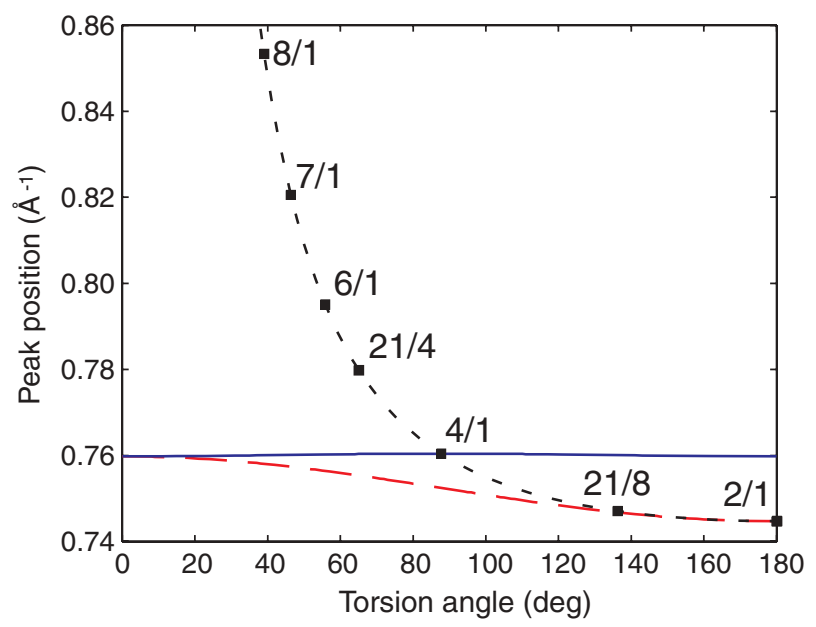

FIG. 3. (Color online) Theoretically predicted position of the most prominent $00 l \mathrm{x}$-ray reflection as a function of torsion angle for dotted black line and square markers: various helical structures. Solid blue line: nonhelical structures where the base unit consists of two or dashed red line: four alternating torsion angles. The two curves show possible nonhelical arrangements that are consistent with the observed periodicity of four monomers in crystals of linear polyfluorenes, e.g., PF8. See text for details. 
necessarily manifest a hexagonal unit cell. For instance, the 4/1 helix should form a tetragonal unit cell [33].

The values for the $(G S)_{2}$ and $(T S)_{2}$ forms do not change significantly in the whole range of values from $0^{\circ}$ to $180^{\circ}$. The torsion angle for a linear side chain polyfluorene, such as PF8, is around $160^{\circ}$ such that the further planarization would have little effect on the peak position. In the ambient conditions, the monomer repeat of PF $2 / 6$ is shorter $(8.06 \AA)$ than the one observed for PF8 $(8.35 \AA)$ and corresponds to the peak position $0.78 \AA^{-1}$. The corresponding peak for PF8 would be hypothetically at $0.755 \AA^{-1}$ but is absent due to the side chain interdigitation. The torsion angle of PF $2 / 6$ is around $65^{\circ}$, and any change in this value would lead to another helical structure (e.g., a 6/1 helix) or loss of periodicity. This is in contrast with the linear polyfluorenes where the proposed structures [Fig. 2] allow planarization to occur while preserving the long range order (chain periodicity). The planarization of PF $2 / 6$ would lead to a much larger change in the monomer period than further planarization of linear polyfluorenes. Moreover, the planarization would lead to the decrease in the monomer period and, thus, the peak shift towards higher scattering angles.

\section{EXPERIMENT}

\section{A. Materials}

The synthesis of PF2/6 [Fig. 1] has been described elsewhere [20]. The number-averaged molecular weight $\left(M_{\mathrm{n}}\right)$ and the weight-averaged molecular weight $\left(M_{\mathrm{w}}\right)$ of the employed polymer were 29.6 and $44.7 \mathrm{~kg} / \mathrm{mol}$, and the polymer was denoted as 30/44-PF2/6. At ambient conditions, this polymer manifests the Hex phase, which transforms into the Nem phase at about $160^{\circ} \mathrm{C}$ [24]. Prior to use, the polymer was annealed at $180^{\circ} \mathrm{C}$ for $10 \mathrm{~min}$ and let to cool down to room temperature.

\section{B. XRD at ambient pressure}

XRD experiments at ambient pressure were performed at the W1.1 beamline at HASYLAB in Hamburg, Germany. A small polymer chip was placed on the substrate, and the experiment was carried out using grazing-incidence geometry. The x-ray energy was $10.5 \mathrm{keV}$, and the sample-to-detector distance was $32.5 \mathrm{~cm}$, yielding a $q$ range of $0.15-1.6 \AA^{-1}$. The beam size was $0.1 \mathrm{~mm}(V) \times 0.2 \mathrm{~mm}(H)$, and the incident angle was $0.14^{\circ}$. Diffraction patterns were measured with an image plate (Molecular Dynamics). In order to suppress radiation damage and scattering from air, a helium atmosphere was employed.

\section{XRD at high quasihydrostatic pressure}

Angle-dispersive XRD experiments at elevated pressures were performed at the Extreme Conditions Beamline (ECB) P02.2 at PETRA III in Hamburg, Germany. The x-ray energy was $25.7 \mathrm{keV}$. The monochromatic $\mathrm{x}$-ray beam was focused at a spot of $4 \mu \mathrm{m}(V) \times 10 \mu \mathrm{m}(H)$ by means of compound refractive beryllium lenses placed $1.2 \mathrm{~m}$ from the sample position. This beam is so narrow that its influence on peak broadening is negligible. The sample-detector distance was $1050 \mathrm{~mm}$, yielding a $q$ range of $0.3-3.5 \AA^{-1}$. Diffraction patterns were measured with a PerkinElmer XRD 1621 amorphous silicon flat panel detector with pixel sizes of $200 \mu \mathrm{m} \times 200 \mu \mathrm{m}$. Images, each acquired with the exposure time of $1 \mathrm{~s}$, were summed up typically 300 times to form one diffraction pattern using the software package QXRD developed at the Advanced Photon Source, Argonne National Laboratory, Chicago. The $q$ range was calibrated using the peak positions of the $\mathrm{CeO}_{2}$ standard.

High pressure was generated in a DAC equipped with anvils pressing a rhenium gasket with a hole that served as a pressure chamber for the sample. The DAC used a conventional diamond upstream and a perforated diamond downstream of the sample. This arrangement is optimal for reducing the Compton scattering background from the DAC [34]. The diamond culets were $0.3-0.4 \mathrm{~mm}$ in size, which enabled pressures over $20 \mathrm{GPa}$. The gasket was indented by the diamonds to a thickness of $0.03 \mathrm{~mm}$, and the gasket hole of $0.15-0.2 \mathrm{~mm}$ in diameter was drilled by an electrical discharge machine. The indented part of the gasket was then placed on the culet of one diamond. The sample and two to three ruby spheres were loaded in the drilled hole such that they did not fill the hole entirely.

In order to provide quasihydrostatic pressure, neon was used as a pressure transmitting medium. The cell was loosely closed after filling the sample and ruby spheres. Neon was loaded at $0.14 \mathrm{GPa}$ using a commercial gas loader (Sanchez Technology, Inc.). The cell, subsequently, was closed at about $1 \mathrm{GPa}$. The pressure was measured by the ruby fluorescence pressure scale [35].

The pressures were increased up to $10 \mathrm{GPa}$, which meant that the background difference due to the thinning of the gasket and change in the diameter of the pressure hole upon pressure increase was considered negligible. The x-ray scattering from empty cells at ambient pressure was measured separately for each employed DAC, and these data were used as a base for background correction.

The absorption and background scattering due to the DAC is position dependent. Therefore, the background curve was scaled individually for each measurement by fitting the background at $q$ values where no peaks were expected between ambient 100 and 110 reflections as well as 200 and 0021 reflections [24]. Bad detector pixels were removed by applying a median or statistical filter piecewise to the two-dimensional data. For each $50 \times 50$ pixel piece, we calculated the statistical average and variance $\left(\sigma^{2}\right)$ based on the $65 \%$ median values and rejected all data points outside the $\pm 5 \sigma$ window.

\section{PL at high quasihydrostatic pressure}

In light of the high-pressure XRD studies of PF2/6, we reanalyzed some of our optical spectroscopic data from PF $2 / 6$ under pressure. The molecular weight of the sample from which the optical spectra was measured was similar to the XRD studies. The PL measurements were taken from the polymer film, which was prepared by drop casting PF2/6 dissolved in dichloromethane onto the surface bottom diamond of the DAC. The cryogenically loaded argon was used as a pressure transmitting medium. Details of the experimental conditions and loading of the DAC are described in Ref. [26]. 


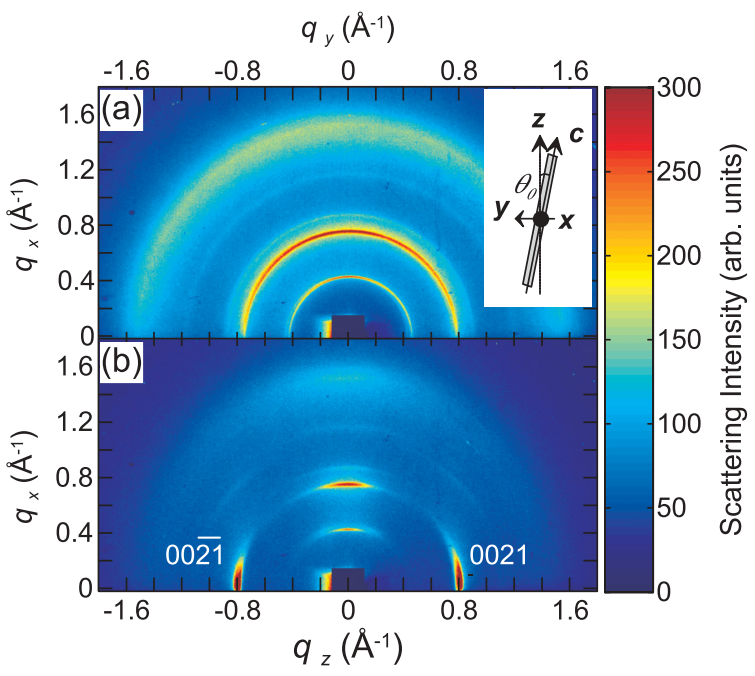

FIG. 4. (Color online) Grazing-incidence XRD patterns of uniaxially aligned 30/44-PF2/6. (a) Incident beam along the $\mathbf{z}$ axis coinciding with the alignment direction. (b) Incident beam along the $\mathbf{y}$ axis. The inset shows the relation between the axes defining the experimental setup ( $\mathbf{x}, \mathbf{y}$, and $\mathbf{z}$ ) and the $\mathbf{c}$ axis coinciding with the approximately rodlike polymer backbone.

\section{RESULTS AND DISCUSSION}

\section{A. XRD at ambient pressure}

Figure 4 shows the XRD patterns of 30/44-PF2/6 at ambient pressure. In order to resolve prominent Bragg reflections better, the polymer sample was annealed, was shear aligned on the glass substrate, and was measured in the grazing-incidence geometry in two directions, parallel and perpendicular to the alignment direction (z). Main hexagonal reflections are visible in Fig. 4(a) and are located perpendicular to the $\mathbf{z}$ axis, whereas, 0021 and its counterpart are conspicuous in Fig. 4(b) and are located along the $\mathbf{z}$ axis. Details of these settings are given in Ref. [36]. This polymer batch is exactly the same and, thus, experienced the same annealing as the polymer employed in the high-pressure XRD experiment. However, when the polymer grains are placed in the DAC, they become randomly oriented with respect to the incoming beam.

\section{B. XRD at high quasihydrostatic pressure}

Figure 5 shows the XRD data for 30/44-PF2/6 around the $q$ range where the predicted $00 l$ peak is appearing. The quasihydrostatic pressure was increased from about 1 to $10 \mathrm{GPa}$. The data show a distinctive peak at $\sim 0.82 \AA^{-1}$ and a shoulder at $\sim 0.86 \AA^{-1}$, which are indexed as $00 l$ and $h 00$. At ambient conditions, these peaks correspond to 0021 and 200. Also plotted are fits to the data, assuming an appearance of two distinct peaks. The relative peak heights dropped with increasing pressure (Fig. 6), but for these data, the fitting was still possible until $\sim 7 \mathrm{GPa}$.

Figure 6 plots the peak heights with increasing pressure for the curves shown in Fig. 5. The height of the $00 l$ reflection is fairly constant until 2 to $3 \mathrm{GPa}$. This is followed by a monotonous decay. Qualitatively similar behavior was reported in our previous paper where the decay began at

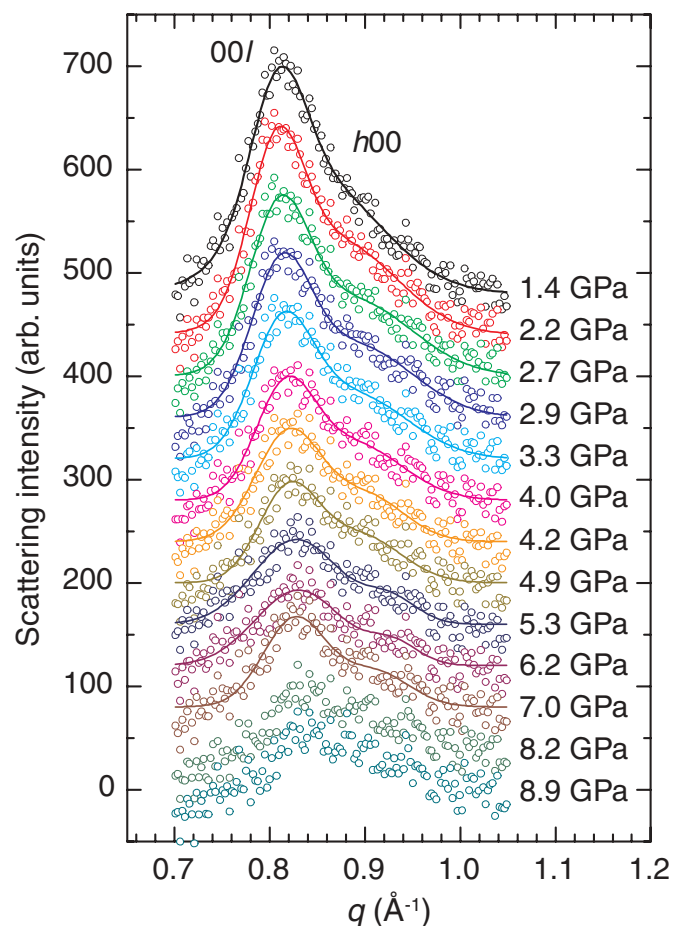

FIG. 5. (Color online) Open small circles: powder x-ray scattering data of the 30/44-PF2/6 polymer with increasing quasihydrostatic pressure at $q=0.7-1.05 \AA^{-1}$. Solid lines: A two-peak scattering model with assumed $00 l$ and $h 00$ reflections fitted to the data corresponding to the 0021 and 200 reflections at ambient conditions. The curves are staggered for clarity.

marginally higher pressures at about $4 \mathrm{GPa}$ [27]. For the most part, the $h 00$ reflection follows the $00 l$ reflection.

Figure 7 plots the fitted peak positions and widths with increasing pressure for the curves shown in Fig. 5. The $00 l$ reflection shifts from 0.81 to $0.83 \AA^{-1}$ between 1 and

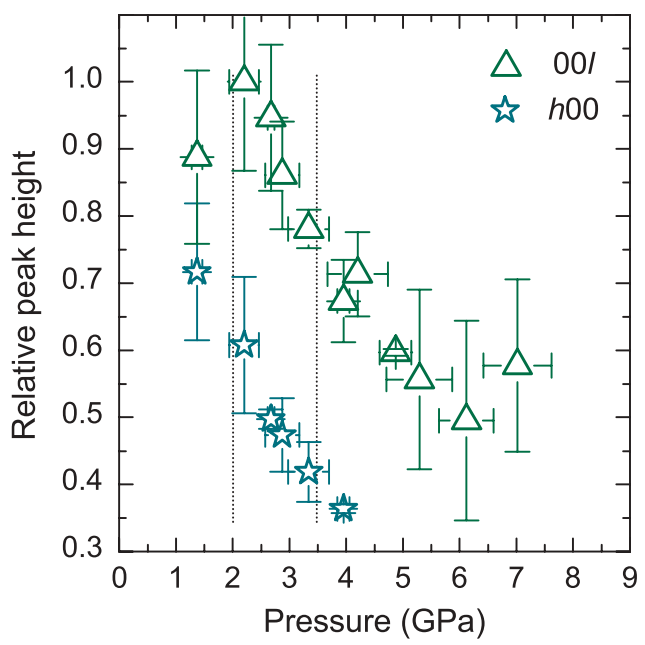

FIG. 6. (Color online) Peak heights calculated from the model fits shown in Fig. 5. The fits correspond to open green triangles and open cyan stars, respectively: the assumed $00 l$ and $h 00$ reflections in the two-peak model for 30/44-PF2/6. Dashed lines mark the position of the optical changes reported by Martin et al. [26]. 

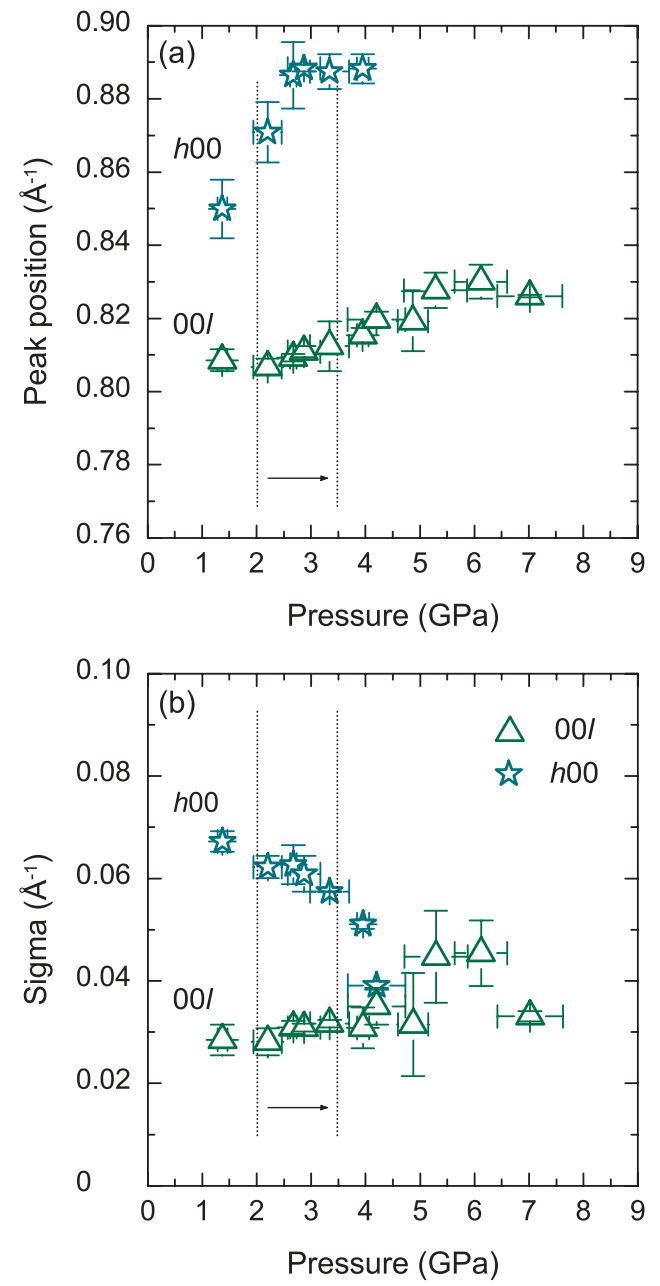

FIG. 7. (Color online) (a) Peak positions and (b) peak width (standard deviation of the Gaussian peak profile) obtained from the model fits shown in Fig. 5. The fits correspond to open green triangles and open cyan stars, respectively: the $00 l$ and $h 00$ reflections in the two-peak model for 30/44-PF2/6. Dashed lines mark the position of the optical changes reported by Martin et al. [26].

$4 \mathrm{GPa}$ and remains fairly constant after this. The peak becomes broader with increasing pressure. At the same time, the $h 00$ reflection shifts from 0.84 to $0.89 \AA^{-1}$, and the peak is actually sharpened.

These data should be assessed against our previously presented ideas of a Hex-Nem like transition [27] and the above-described model of main chain planarization as well as against the observed optical transition and planarization idea of Guha and Chandrasekhar [25] and Martin et al. [26].

We can rationalize the similarities and differences between our previous paper [27] and the data shown in Fig. 5 as follows. In both cases, the $\mathrm{x}$-ray reflections are weak, and we are able to observe and to identify 0021 and, occasionally, 200 reflections. In both cases, the 0021 peak is significantly broadened, and this, with the loss or significant broadening of $h k 0$ peaks, implies a morphological transition from the crystalline Hex phase to a weakly ordered phase. In the previous paper, we proposed that the high-pressure structure resembles an ambient glassy Nem phase.
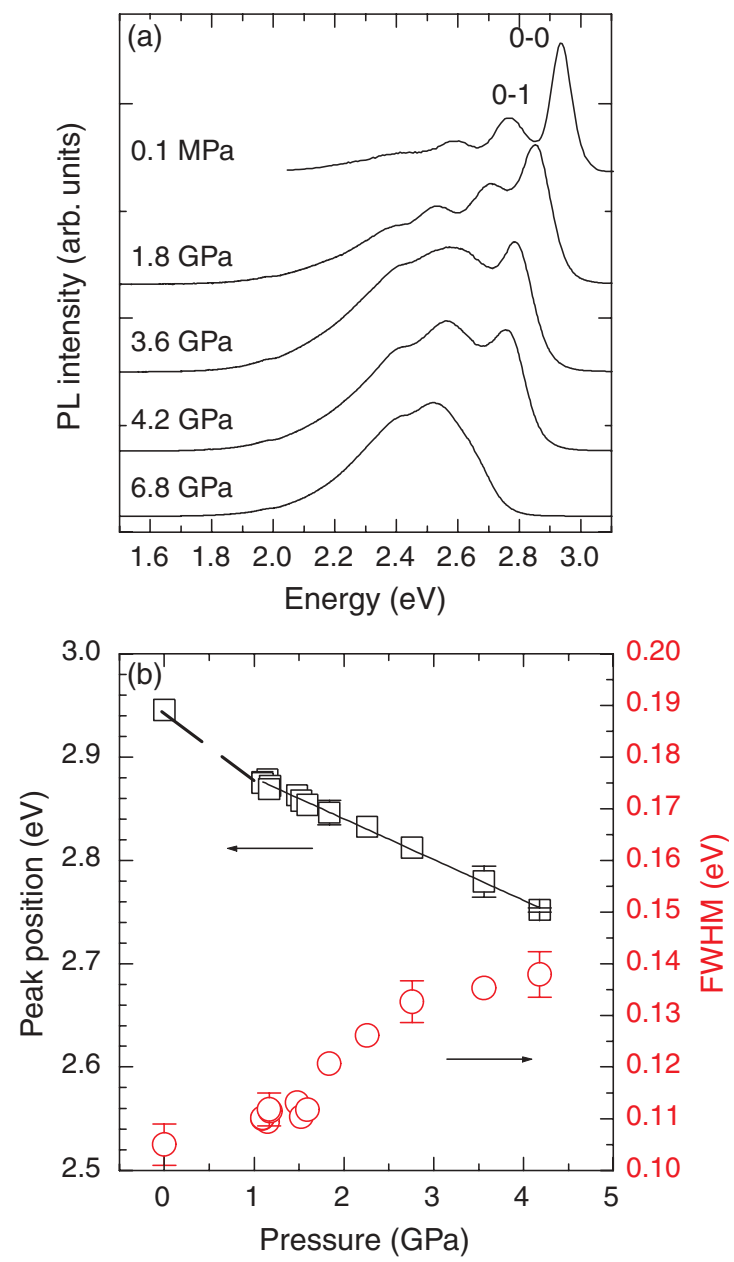

FIG. 8. (Color online) (a) PL spectra of a PF2/6 film at selected values of pressure. (b) Open black squares: the peak position and open red circles: the FWHM of the $0-0$ peak with increasing pressure. The solid line is a linear fit. The dashed line is a guide to the eye.

In the previous paper [27], the observed changes in the XRD data were sharp. In the present case, the same changes are observed, but no prominent transition exists. In both cases, the intensity of the 0021 peak drops, and the peak moves towards higher scattering angles, the latter fact being consistent with the partial planarization of the main chain. In the earlier case, we observed the peak shift back to the lower angles, whereas, in the present case, this is either not visible or very weakly visible [Fig. 7(a)]. Possible reasons explaining this difference are different pressure transmitting media (ethanol-methanol versus neon), different compression rates, or radiation damage.

We can rationalize the experimental peak position [Fig. 7(a)] against the theoretically predicted position [Fig. 3] as follows. Figure 3 plots the theoretically predicted position of the most prominent $00 \mathrm{l}$ reflection as a function of torsion angle. This shows helical conformations (dotted black line) with some periodic helices marked by symbols. The hypothetical fully planar backbone is represented as the $2 / 1$ conformation, and this corresponds to the lowest peak position. At ambient pressure, the 0021 reflection of crystalline PF2/6 occurs at $0.78 \AA^{-1}$, which exactly matches the $21 / 4$ helical backbone conformation with the torsion angle of $\sim 65^{\circ}$ [21]. 
Theoretically, the peak position increases towards higher scattering angles when moving from the 21/4 helix towards other more planar helices (such as the 6/1 helix) [Fig. 3]. Experimentally, the $00 l$ reflection occurs at $0.81 \AA^{-1}$, which is higher than in the crystalline PF2/6 in the ambient conditions, even if extrapolated to zero pressure [Fig. 7(a)]. This means that the model constructed above is in accordance with the more open helix and explains the observed smaller repeat distance (higher peak position), even though we cannot determine the exact main chain configuration among the presented configuration family.

Figure 7(b) plots the width of the $00 l$ reflection with increasing pressure. This value [full width at half maximum $\left.(\mathrm{FWHM})=2.35 \sigma=0.064 \AA^{-1}\right]$ is significantly larger in the higher pressures than in the crystalline state at the ambient pressure $\left(\mathrm{FWHM}=0.02 \AA^{-1}\right.$ [24]). This implies that, at the elevated pressures, the material is weakly ordered rather than crystalline, potentially already at $\sim 1.5 \mathrm{GPa}$. This weakly ordered structure resembles ambient Nem phases. At ambient pressures, the peak position at $0.81 \AA^{-1}$ is typical for the weakly ordered Nem phase rather than the crystalline Hex phase, whether that of a LMW-PF2/6 at room temperature [24] or that of a HMW-PF2/6 above the Hex-Nem transition [21]. Referring to Fig. 3, the nematic state corresponds to a more open helix (smaller torsion, i.e., more towards planar cis conformation).

In our previous paper [27], we presented a scenario where the intensity of the 0021 peak dropped with the simultaneous peak shift plus the disappearance of $h k 0$ peaks, and these changes marked the pressure induced Hex-Nem transition at $\sim 2-4 \mathrm{GPa}$. In the present case, we observe similar changes in data but no sharp transition. We now put forward another scenario where the pressure induced transition from the crystalline to the weakly ordered state has taken place earlier than previously expected, and even the first measured pressure of about $1.5 \mathrm{GPa}$ may already represent the pressure induced weakly ordered state.

The nature of the high-pressure structure compared to the ambient nematic phases is not clear. We can only observe the 200 peak, i.e., 100 and 110 are clearly absent. In the crystalline material, 100 and 110 should be stronger than 200. Nem HMWPF2/ 6 shows only the 110 peak, and Nem LMW-PF2/6 shows all the reflections as in the crystalline material but significantly broader and merged. The fact that the 110 reflection is absent may indicate that the pressure induced state has lost its all hexagonal packing symmetry that is weakly present for both ambient nematic materials.

\section{PL at high quasihydrostatic pressures}

As pointed out earlier, a redshift in the PL or absorption energy with increasing pressure reflects both higher conjugation as well as planarization of the backbone chain, especially for those $\pi$-conjugated molecules and polymers that have nonplanar backbone conformations at ambient conditions. The additional redshift, due to planarization of the backbone, typically shows up as a nonlinear shift in the PL energies as observed, for example, for PF8 [12] and parahexaphenyl [37].

Another indicator of the planarization of the backbone chain is to compare the relative ratio of the $\mathrm{C}-\mathrm{C}$ backbone stretch mode to the $\mathrm{C}-\mathrm{H}$ bend mode in the Raman spectra. This methodology has been useful in observing the planarization of the backbone in parahexaphenyl [37] and other smaller oligophenyls under pressure. Unfortunately, the $\mathrm{C}-\mathrm{C}$ backbone stretch modes in the PFs lie around $1300 \mathrm{~cm}^{-1}$, right at the position of the strong $1330 \mathrm{~cm}^{-1}$ diamond Raman vibration from the DAC. This completely overwhelms the Raman signature of PF samples in this frequency region.

In light of the above-described XRD data, we reanalyzed some of our optical spectroscopic data from PF2/6 under pressure. Figure 8(a) shows the PL spectra of a PF2/6 film at a few selected pressures, including the data for the ambient condition. Figure 8(b) shows the peak position and the FWHM of the 0-0 vibronic peak. In these experiments, the lowest pressure in the DAC was $\sim 1 \mathrm{GPa}$, thus, corresponding to the XRD data shown in Fig. 5.

The PL emission of PFs shows a well-resolved FranckCondon type progression of vibronic sub-bands in addition to the $\pi-\pi *$ electronic transition. The emissive transition highest in energy is the 0-0 transition. Although the data shown here are for the 0-0 PL vibronic peak, the other vibronic peaks show a similar behavior with increasing pressure.

The PL energy redshifts linearly beyond $1 \mathrm{GPa}$, but the FWHM shows a discontinuity around $2.7 \mathrm{GPa}$. The PL linewidth under pressure is an indicator of enhanced interchain interactions [12]. The discontinuity in the 0-0 PL FWHM at $\sim 2.7 \mathrm{GPa}$ could reflect a transition to the weakly ordered state with a concomitant planarization of the backbone.

There is a slight hint of nonlinearity between the ambient condition $0-0$ vibronic peak position and the $1 \mathrm{GPa}$ data [Fig. 8(b)] as represented by the dotted line, which could be an indication of a planarization of the backbone chain already below $\sim 1 \mathrm{GPa}$. This is comparable to the above presented scenario where the pressure induced Hex-Nem transition could take place earlier than previously expected, even below 1.5 GPa. Such nonlinearity in the PL peak positions are pronounced in small molecules around 1.5 GPa [37]. Unfortunately, tuning the pressure with a conventional DAC below $1 \mathrm{GPa}$ is difficult, and we could not measure optical data in this low-pressure regime.

\section{CONCLUSIONS}

We have conducted an x-ray scattering study of PF2/6 under quasihydrostatic pressure from 1 to $10 \mathrm{GPa}$ at room temperature. First, we constructed a molecular model for a polyfluorene backbone, considered the torsion angle between repeat units, and calculated the position of the most prominent $\mathrm{X}$-ray reflection in the [00l] direction. Second, we identified and followed the experimental $00 l$ reflection and compared its position to the theoretical peak position. The employed PF2/6 is crystalline and shows a hexagonal unit cell at ambient pressure. The width of the $00 l$ peak is significantly increased with increasing pressure, which indicates a smooth structural transformation from this crystalline structure towards a weakly ordered structure. This transformation appears more gradual and seems to begin at lower pressures (at or below $2 \mathrm{GPa}$ ) than previously expected ( $\sim 4 \mathrm{GPa})$. The intensity of the $00 l$ peak drops, and it moves towards higher scattering angles with increasing pressure, which, together with the given 
molecular model, point to the planarization of the helical main chain. However, the previously observed move back towards lower angles was not observed. The reason is not known, but it may be a possible effect between presently and previously used pressure transmitting media (a neon versus a methanol-ethanol mixture) or radiation damage. Third, we revisited the previously collected PL data also pointing to the enhanced interchain interactions and main chain planarization.

The question of the previously proposed intermolecular Hex-Nem phase transition with increasing pressure may be open. In order to clarity this issue, future papers should focus on the low molecular weight PF2/6, which appears nematic at ambient pressure. Future papers should also contain systematic screening and optimization of experimental conditions. This would lead to a better signal-to-noise ratio, ideally, the unit cell parameters as a function of pressure. It would, moreover, reveal possible experimental artifacts.

This paper gives an example of how a helical $\pi$-conjugated backbone can be controlled by high quasihydrostatic pressure without chemical modifications to the polymer itself. Optical spectroscopic studies under hydrostatic pressure have been conducted for many $\pi$-conjugated polymers for more than two decades. A lack of XRD studies has been a bottleneck for mapping their full behavior as a function of pressure driven intra- and interchain interactions. We believe that XRD studies become increasingly important in understanding their optical high-pressure properties and possibly the structure-property relationships in general.

\section{ACKNOWLEDGMENTS}

We thank W. Caliebe of HASYLAB for help with the experiment at the $\mathrm{W} 1$ beamline. The synchrotron experiments were supported by the European Community's Seventh Framework Programme (FP7/2007-2013) under Grant Agreement No. 226716. Part of this project was supported by the PETRA III sample environment group. S.G. thanks C. M. Martin for the PL data and M. Chandrasekhar for access to her research facility and useful discussions.
[1] V. Marcon, N. van der Vegt, G. Wegner, and G. Raos, J. Phys. Chem. B 110, 5253 (2006).

[2] S. Ko, E. T. Hoke, L. Pandey, S. Hong, R. Mondal, C. Risko, Y. Yi, R. Noriega, M. D. McGehee, J.-L. Brédas, A. Salleo, and Z. Bao, J. Am. Chem. Soc. 134, 5222 (2012).

[3] G. Mao, M. J. Winokur, and F. E. Karasz, Phys. Rev. B 58, 4089 (1998).

[4] E. Da Como, N. J. Borys, P. Strohriegl, M. J. Walter, and J. M. Lupton, J. Am. Chem. Soc. 133, 3690 (2011).

[5] M. Chandrasekhar, S. Guha, and W. Graupner, Adv. Mater. 13, 613 (2001).

[6] J. P. Schmidtke, R. H. Friend, and C. Silva, Phys. Rev. Lett. 100, 157401 (2008).

[7] J. P. Schmidtke, J.-S. Kim, J. Gierschner, C. Silva, and R. H. Friend, Phys. Rev. Lett. 99, 167401 (2007).

[8] S. Albert-Seifried, J. M. Hodgkiss, F. Laquai, H. A. Bronstein, C. K. Williams, and R. H. Friend, Phys. Rev. Lett. 105, 195501 (2010).

[9] Y.-S. Huang, J. Gierschner, J. P. Schmidtke, R. H. Friend, and D. Beljonne, Phys. Rev. B 84, 205311 (2011).

[10] V. Morandi, M. Galli, F. Marabelli, and D. Comoretto, Phys. Rev. B 79, 045202 (2009).

[11] K. Paudel, M. Arif, M. Chandrasekhar, and S. Guha, Phys. Status Solidi B 246, 563 (2009).

[12] K. Paudel, H. Knoll, M. Chandrasekhar, and S. Guha, J. Phys. Chem. A 114, 4680 (2010).

[13] A. Katrusiak, Acta Crystallogr., Sect. A: Found. Crystallogr. A 64, 135 (2008).

[14] G. Heimel, P. Puschnig, M. Oehzelt, K. Hummer, B. Koppelhuber-Bitschnau, F. Porsch, C. Ambrosch-Draxl, and R. Resel, J. Phys.: Condens. Matter 15, 3375 (2003).

[15] M. Oehzelt, G. Heimel, R. Resel, P. Puschnig, K. Hummer, C. Ambrosch-Draxl, K. Takemura, and A. Nakayama, J. Chem. Phys. 119, 1078 (2003).

[16] G. Heimel, K. Hummer, C. Ambrosch-Draxl, W. Chunwachirasiri, M. J. Winokur, M. Hanfland, M. Oehzelt, A. Aichholzer, and R. Resel, Phys. Rev. B 73, 024109 (2006).
[17] J. Mårdalen, Y. Cerenius, and P. Häggkvist, J. Phys.: Condens. Matter 7, 3501 (1995).

[18] J. Mårdalen, E. J. Samuelsen, O. R. Konestabo, M. Hanfland, and M. Lorenzen, J. Phys.: Condens. Matter 10, 7145 (1998).

[19] J. Corish, D. A. Morton-Blake, F. Bénière, and M. Lantoine, J. Chem. Soc., Faraday Trans. 92, 671 (1996).

[20] M. Grell, W. Knoll, D. Lupo, A. Meisel, T. Miteva, D. Neher, H.-G. Nothofer, U. Scherf, and A. Yasuda, Adv. Mater. 11, 671 (1999).

[21] M. Knaapila, M. Torkkeli, and A. P. Monkman, Macromolecules 40, 3610 (2007).

[22] M. Brinkmann, N. Charoenthai, R. Traiphol, P. Piyakulawat, J. Wlosnewski, and U. Asawapirom, Macromolecules 42, 8298 (2009).

[23] B. Tanto, S. Guha, C. M. Martin, U. Scherf, and M. J. Winokur, Macromolecules 37, 9438 (2004).

[24] M. Knaapila, R. Stepanyan, M. Torkkeli, B. P. Lyons, T. P. Ikonen, L. Almásy, J. P. Foreman, R. Serimaa, R. Güntner, U. Scherf, and A. P. Monkman, Phys. Rev. E 71, 041802 (2005).

[25] S. Guha and M. Chandrasekhar, Phys. Status Solidi B 241, 3318 (2004).

[26] C. M. Martin, S. Guha, M. Chandrasekhar, H. R. Chandrasekhar, R. Guentner, P. Scanduicci de Freitas, and U. Scherf, Phys. Rev. B 68, 115203 (2003).

[27] M. Knaapila, R. Stepanyan, D. Haase, S. Carlson, M. Torkkeli, Y. Cerenius, U. Scherf, and S. Guha, Phys. Rev. E 82, 051803 (2010).

[28] G. Lieser, M. Oda, T. Miteva, A. Meisel, H.-G. Nothofer, U. Scherf, and D. Neher, Macromolecules 33, 4490 (2000).

[29] A. Gitsas, G. Floudas, and G. Wegner, Phys. Rev. E 69, 041802 (2004).

[30] S. H. Chen, A. C. Su, C. H. Su, and S. A. Chen, J. Phys. Chem. B 110, 4007 (2006).

[31] M. Grell, D. D. C. Bradley, G. Ungar, J. Hill, and K. S. Whitehead, Macromolecules 32, 5810 (1999).

[32] S. McFarlane, R. McDonald, and J. G. C. Veinot, Acta Crystallogr., Sect. C: Cryst. Struct. Commun. C 61, o671 (2005). 
[33] H. Tadokoro, Structure of Crystalline Polymers (Wiley, New York, 1979).

[34] E. Soignard, C. J. Benmore, and J. L. Yarger, Rev. Sci. Instrum. 81, 035110 (2010).

[35] P. M. Bell, H. K. Mao, and K. Goettel, Science 226, 542 (1984).
[36] M. Knaapila, R. Stepanyan, B. P. Lyons, M. Torkkeli, and A. P. Monkman, Adv. Funct. Mater. 16, 599 (2006).

[37] S. Guha, W. Graupner, R. Resel, M. Chandrasekhar, H. R. Chandrasekhar, R. Glaser, and G. Leising, Phys. Rev. Lett. 82, 3625 (1999). 